DOI: 10.14526/2070-4798-2019-14-4-22-31

\title{
Chi Kung in the structure of modern physical culture and sports activity
}

\author{
Natalya Yu. Tarabrina*, Yuriy V. Kraev \\ Moscow Aviation Institute (National Research University) \\ Moscow, Russia \\ ORCID: oooo-ooo3-1469-501o, nata-tarabrina@mail.ru* \\ ORCID: OOOO-OOO2-2034-3938, yury.kraev@mail.ru \\ Review article
}

\begin{abstract}
One of the most precious heritages of Chinese medicine, that has survived, is Chi Kung system (method). It is a scientific, therapeutic, preventive, creative and military means of human development. Materials and methods. Special importance this system has in difficult and chronic diseases therapy. In this connection in ancient times Chi Kung (therapy) was called "the method of diseases eradication and life support". Results. The article presents short historical information and the characteristics of a traditional health-improving Chi Kung system, its theoretical-methodological analysis; general review of the exercises and the system of psycho-corporal training; the demands, claimed on nutrition and clothes; the basic principles of Chi Kung, individual features, time and ways of lessons. Conclusion. It is shown that Chi Kung is a system of teaching. It helps to use natural abilities of body and consciousness for personal development, both in terms of health improvement and spiritual and creative abilities development.
\end{abstract}

Keywords: Chi Kung, History, theory, methodology, exercises, physical culture.

For citation: Natalya Yu. Tarabrina*, Yuriy V. Kraev. Chi Kung in the structure of modern physical culture and sports activity. Russian Journal of Physical Education and Sport. 2019; 14(4): 19-26. DOI: 10.14526/2070-4798-2019-14-4-22-31.

\section{INTRODUCTION}

The system of Taoistic Chi Kung gymnastics is the quintessence of the ancient Chinese scientists valuable experience,. They studied the problems of health preservation and lifetime increase [1]. Special importance this system has in difficult and chronic diseases therapy. In this connection in ancient times Chi Kung (therapy) was called "the method of diseases eradication and life support". The system of the traditional Chinese Chi Kungtherapy was formed during many centuries in terms of labor activity and the struggle of ancient Chinese people with different diseases [2]. In ancient times Chi Kung had different names: management Chi (Dao-yin), expiration - collecting (Tu-na), energy training (Lyan-chi), quiet sitting (Tsin-tso), sitting meditation (Tso-chan), inner work (Nei-gun), the art of inner elixir (Nei-dan-shu) and others[3].

Information about Chi Kung can be found in many written monuments of Chinese civilization. They were written several thousand years ago [3]. In a well-known tractate "Neyztsiz" - "Inner diseases" - great attention is paid to Taoistic respiratory gymnastics "Dao-yin" among other therapeutic means. In another medical tractate different therapeutic means are enumerated: “...Taoistic respiratory gymnastics "Dao-yin", therapeutic massage, cauterization, rubbing in a hot medicinal preparation, acupuncture, medicine ingestion...”. During khan graves of Ma Vanyutu excavation among other written monuments of Chinese ancientry there was the scheme of treatment using Taoistic respiratory gymnastics. This scheme had not only hieroglyphic explanations, but also graphic illustrations. It proves that during Chunytsyu period (770-476 B.C.) and during Khan dynasty (IIIII century AD) Taoistic gymnastics was widely used [4].

In ancient times Taoistic respiratory gymnastics "Dao-yin" was used as one of effective means of struggle against different diseases. In comparison with other therapeutic agents respiratory gymnastics had a range of significant advantages: during the process needles were not used, pharmaceutical drugs were not used, which was very convenient and efficient [5]. It was important that respiratory gymnastics use was completely painless. The most valuable thing was that Taoistic 
respiratory gymnastics "Dao-yin" was directed toward all organism treatment and strengthening. If a person was ill this gymnastics helped to cope with the disease, if a person was healthy it was a great prevention means and provided long life of people. All known medical men in ancient China paid great attention to Chi Kung-therapy.

The founders of Taoism is Lao-tzu, the author of the tractate Dao de tzin (VI century ACN) and the teacher of Chzhuantzu, who lived three centuries after that. He is the author of Taoism tractate [3,4]. Chinese doctor of Khan dynasty (947-950 AD) Khua Ta, summarizing practical experience of own predecessors, combined all exercises of Taoistic respiratory gymnastics "Dao-yin" and divided then into 5 types, which he called "Five units". Apart from that Khua Ta gave theoretical substantiation to all mentioned above five kinds. Khua Ta analyzed the methodology of Taoistic respiratory gymnastics and created the system of physical exercises which imitated the movements of a tiger, a deer, a bear, a monkey and a stork. A new system of exercises was widely used. There was a great number of longlivers among people, who practiced this system. The followers of Khua Ta - U Tsu, Fan A and some others, taking as the base the methodology of their teacher, lived more than 90 years and even at this age they had good health state, they had perfect auditory sense and eyesight, they had all teeth safe $[3,4]$.

Nowadays the methodology of physical exercises Chi Kung is used in Japan and Korea, America and Europe. More than 2 million people go in for respiratory gymnastics in other countries $[6,7]$. Great attention abroad is paid to studying the aspects of Chi Kung-therapy [8]. These research works are held according to two main directions:

1) observation with the help of modern scientific-technical means over physical and physiological effects. They define people, who go in for Chi Kung-therapy, from other people [9];

2) observation over the people, who go in for Chi Kung-therapy in terms of clinical conditions.

Well-known Chinese medical men Ge Khun, Tao Khuntsizni, Chao Yuanfon, Sun Symyao, Li shitsai, Van An, Shen Tsinao and others described different methods of the traditional Taoistic respiratory gymnastics [3].

Later the ruling feudal classes made all efforts to make this means unavailable for simple people and for further generations. This way Chi Kung started to be unavailable for people. Chi Kungtherapy turned into a health improving means for the chosen classes and Taoistic monks. They secretly practiced the corresponding exercises.

The system of Chi Kung-therapy includes 5 methodologies of Taoistic gymnastics: "daoyin""yinyan" - "idea", "khusi"- "breathing", "tyaosi" "breathing regulation", "shitsze" - "power", "tuin" - "sound", "anbmo" - "massage" [1,2].

In an old system of Chi Kung usually 4 positions were used in order to fulfill the exercises - "standing, sitting, lying, standing on knees". In a new Chi Kung system these positions form the base. They are added by all advantages of " 5 imitations" of Khua-Ta. Thus, the authors developed the old Chi Kung system, such basic directions of Taoistic respiratory gymnastics "Dao-yin", as "idea", "breathing", "power" and others, making them the base of "dynamism" concept. Thus, in its transformed and added kind a new Chi Kung gymnastics includes more than 22 "dynamic" methodologies for different diseases treatment and prevention. In a new kind Chi Kung therapy combines the effectiveness of two kinds of gymnastics - "Dao-yin" and "Sin-tsi". It is simultaneously a movement and rest (rest in movement and movement at rest). Preserving the main base of Chi Kung, the authors combined inner and outer movements, such notions of respiratory gymnastics as "idea", "breathing" and "power".

In ancient times Taoistic respiratory gymnastics "Dao-yin" was an integral system, where two kinds of Taoistic respiratory gymnastics were connected. That is why the ancient initial Chi Kung system was able to have full health-improving effect. However then appeared people, who started to explain "Dao-yin" methods as a simple system of physical exercises and "Sin-tsi" methodology was considered as something out-of-date. Thus, there was two interconnected forms of "Dao-yin" division. There were also a lot of people, who thought that "though with the help of Chi Kung it is possible to stop the disease for a long time period. But still there are a lot of not clear moments". They presented Chi 
Kung as something that "can give hope, but is not available" [3].

A new Chi Kung therapy changed the ways and methodologies of two separate existing schools, static and dynamic. It recovered ancient methods and developed them gradually. In a new system main attention is paid to a close dynamic unity of "idea", "breathing" and "power" from "Dao-yin" gymnastics; the combining initial unit in this case is "idea", as on the degree of mastering the "idea" depended the amount of the reproduced "inner air" and the degree of health-improving influence on the disease. In order to give patients, who train according to this system, the opportunity to combine "idea", "breathing" and "power", special attention was paid to "three stage, connecting the separate" method creation $[1,10]$.

It means that at the initial stage of trainings the activity of the "idea" should be directed toward the top of "power" achievement. After gaining the skills of "power" in terms of trainings increases the activity of "idea", not clear moments become clear. Starting from this stage, the activity of "idea" should be concentrated and lifted till the level of "Te". "Te" is an imaginary concentration of "idea" activity, something material, seen or something that can be expressed by some number - so there is no misunderstanding in the exercise fulfillment.

The following things can be fulfilled: "idea" activity, initially concentrated on some outer subject or a thing, transfer and concentrate in some point of inner body (usually "idea" concentration is realized in the average point of Dan-tyan) [11].

Observing such kind of succession in mastering exercises favorable opportunity is created for a gradual transfer from the training process mastering for "idea" control in the system of "Dao-yin" to the ability to control the energy of "Dan-tyan" points. Without observing mentioned above conditions it is impossible to teach a person to control the energy in "Dan-tyan" points [11].

The difficulties of the initial stage of trainings are in the fact that it is impossible to make the activity of "idea" lower. It is necessary to try to make "idea" concentration stronger. The activity of "idea" increase can cause headache, dizziness, slight asthma, abdominal distention and other unpleasant states. That is why if we observe "Tyao-se" rule ("coordinated breathing") according to ancient Chi Kung methodology, it is necessary to give the activity of "idea" respiratory base at the beginning of the training process. During the trainings the phenomenon of the intensified breathing would take place, till the sense of asthma or other not comfortable senses. Only after the activity of "idea" wouldn't be restricted during the breathing act, the process of respiration would set and become even and calm.

Some changes introduction into the ancient Chi Kung system helps to master easily the complex of this gymnastics new methodology $[1,2,3]$. Exercises fulfillment should be realized in the definite order and gradually. It is not recommended to have deviations, only this way maximum healthimproving effect can be provided. Among most people, who train according to a new Chi Kung system, "idea" has an even and calm state, "power" is free, uninhibited and relaxed, breathing becomes even and calm [5].

Let's consider the definite ways of mastering 6 main methodologies of a health-improving gymnastics: even "fen" breathing during normal walking; "fen" breathing with the set amount of steps; natural breathing in terms of a slow walking; not big sticks spinning in hands; massage of "yutsyuan" points; massage of the points on the head.

All these methodologies can be used as the initial treatment for most diseases [9,12]. It is obvious that the statement about the fact that the methodology of a traditional Chinese Chi Kung therapy is an effective means in many diseases treatment, doesn't mean that this system can do everything. Not great effectiveness of a new methodology goes together with traditional means and is demonstrated together with traditional means and methods of Chinese and European medicine $[13,14]$.At the same time it should be noted that the methodology of Chi Kung gymnastics also needs further development and improvement.

General review of the exercises. System of psycho-corporal readiness

Before starting to train a person should be sure 
that Chi Kung gymnastics would be able to change his physical, functional and spiritual state. Moral training, equipment are necessary. The time and place of lessons should be defined, a person should give oneself a mindset for long-term trainings. Only being completely confident and sure a person can master the whole complex of exercises and within a short time period achieve the set results $[8,10]$.

Undoubtedly there are differences between the ways of diseases treatment with the help of exercises and with the help of other known methods. They are in the following: diseases treatment with the help of the exercises is realized by means of outer influence on an organism [7,8]. The confidence of a person that he would recover with the help of these exercises is an important part of the recovery. The recovery of a person also depends on a person, who organizes the trainings.

Speaking about diseases treatment with the help of the exercises, the result depends on the ways and methods, offered by the instructors, but finally everything depends on the working capacity of a person, his ability to control own body in order to achieve a steady "yin" and "yan" state in an organism. Owing to this way there appear the opportunities for the influence on the meridians of a human organism. Thus, the more confidence a person has, the greater would be the effect of the exercises. Thus, confidence is the initial condition of a positive effect of the lessons: if a person has self-confidence, then he would have resoluteness. Self-confidence together with resoluteness would become the base for constancy. In this connection there appears the following question: Where should confidence appear from? It can't come from emptiness. A person, who started to master the exercises, would not fully believe Chi Kung methodology, which is natural. But when he feels support and sees the effectiveness of this method, he changes his attitude to lessons. When a person, who trains is able to feel the influence of effective lessons on an organism, it would become the best motivation for him, he would be confident in a new Chi Kung methodology.

The question of confidence strengthening in a positive effect of the gymnastics is also important, it is necessary to accumulate and master the experience of each person, who trains according to this system, systematize the accumulated experience in order to achieve higher rational cognition. Only understanding the verity of this methodology and its theoretical basis, achieving the aim by means of intensive exercises, realizing transfer from sensuality to rational real truth cognition of a positive effect from a new Chi Kung methodology, a person can be sure of its effectiveness.

\section{Demands, claimed on nutrition and clothes}

The best clothes for Chi Kung lessons are soft clothes that don't hinder movements. Trousers shouldn't hinder movements and pull in too tight the waist [15].

During off-season, when the weather changes, corresponding suitable for a season clothes should be worn. In winter attention should be paid to the ability of clothes to keep body warmth, it is necessary to put on hat and warm footwear. A person shouldn't be cold. These rules would provide a normal activity of all organism systems and it would be easier to achieve the state of rest during the exercises fulfillment.

During the exercises fulfillment it is necessary to control the waist. It shouldn't be pulled in too tight, cuffs and collar should be unbuttoned, footwear should be low heeled (it's better to have made of textile fabric footwear).

If the trainings begin early in the morning, a person shouldn't eat. 30 minutes after the training a person can eat a regular breakfast. Before daily and evening trainings a person shouldn't eat a lot. Trainings can be started only 30 minutes after a meal. After having rest after the exercises, a person can eat and start his everyday activity.

It is not recommended to eat peppered and spicy food, onion or garlic can be eaten only after cooking. A person shouldn't smoke and drink alcohol.

The window in the room for trainings should be open in order to provide clean fresh air. In early morning the place should be chosen near water or in the places, where there are pines and cypresses. Moreover, the place for trainings should be even and 7 kinds of interference should be avoided: fun, 
anger, sorrow, thoughts, worry, fear, phobia. Only in this case there would be any effect of the trainings.

Planning the structure and volume of trainings a teacher should take into account the state of a student. Morning trainings should last 2-3 hours, after dinner or in the evening 2 hours. In the morning it is more effective to master natural "fen" breathing on one, two and three steps in the average tempo or slow natural breathing. In the evening students can spin not big sticks in hands; massage key "yutsyuan" points, in the afternoon before a meal head massage can be fulfilled [15].

\section{The main principles of Chi Kung}

\section{gymnastics}

The main principle of Chi Kung therapy is the principle of "roundness, softness, distance", regardless of the kind of exercise a person does. In any case these moments should be followed, it is especially important for the exercises with slow natural breathing $[1,16]$.

Roundness: during exercises fulfillment body movements should be round (or arched), extremities shouldn't be constrained in joints; this way normal "air" and blood circulation would be provided.

Softness: neck, body and extremities muscles and all joints should be relaxed. During movement fulfillment the definite degree of relaxation should be preserved, at the same time, relaxation shouldn't become "carelessness".

Distance: in spite of the fact that eyes should be slightly open, a person should look directly straight forward. Fulfilling the exercise a person should concentrate on some outer thing or sense. Only having gained some experience in mastering the exercises thoughts can be focused on the pace of "Dan-tyan" localization.

During the process of exercises accumulation a person should control own actions, comparing then with "roundness, softness, distance" principle. Only following this principle it is possible to create "inner air", which would provide health-improving effect of the exercises.

"e, tsi, sin" achievement

"E" is the product of "idea" activity. "E" includes the idea, senses, consciousness, thinking. "Ti" is an "inner air". "Sin" is a corporal activity, "power". These three categories are connected with each other. It is known that the aim of the exercises is "inner air" release, creating the state of balance "yin" and "yan", immunity systems strengthening. At the same time, it is necessary to use all ways of Taoistic respiratory gymnastics "Dao-yin", go through three important key points ("calmness", "idea", "coordinated breathing”). Different ways and variants of the exercises fulfillment are connected, so it is forbidden to exclude one of the parts. However, in the system of Taoistic respiratory gymnastics "Dao-yin" "power" and respiration also influence the thought [16]. As the movement of a relaxed soft body has a positive influence on thought relaxation and on calmness achievement in brain activity. The same way a long-term calm breathing influences calm work of brain.

An accurate use of the methods of "idea" activity improvement can lead to the fact that a cortex in the definite conditions would be at rest and we have only to chose the definite complex of exercises, concentrate "idea" on one point or on some object and in this case it is possible to turn on the mechanisms of "air" movement, realizing greater amount of "inner air". As "inner air" release is fulfilled through "idea", in the system of Taoistic respiratory gymnastics "Daoyin" this process is called "E" and releases "Tsi". During the exercises fulfillment apart from "idea" activity it is also necessary to distribute "power"; rational "power" distribution can help in "inner air" production and it would provide movement according to the definite way. Those, who only start their trainings, don't have necessary "power", that is why at the initial stage they should give some time to the questions of "power" mastering. After that "power" would grow and there would be no need to pay great attention to it: by this time released with the help of trainings "inner air" would be able to regulate necessary amount of "power", which in Chi Kung gymnastics is called "Tsi" releases "Sin"” $[16,17]$.

When the beginners master "power" the activity of "idea" is in a concentrated state. During this period brain work shouldn't be directed toward lack of coordination avoidance in thoughts, and think that "power" was influenced by "idea": "E" leads to "Sen" decrease as such thoughts would have a 
negative influence on "inner air" production and the disease can turn into a relapse. During this period it is necessary to choose the definite methodology for "idea" concentration and lead own attempts to "inner air" release. It would help to release "power". At the initial stage of the training lessons connection between "E", "Tsi" and "SIN" categories is restricted by the fact that "E" releases "Tsi" and "Tsi" releases "SIN" [17].

In Chi Kung gymnastics there is also the notion that "E" controls "Tsi", which is the same as the motion "E" releases "TSI". Their difference is in the following: in the notion "E" releases "TSI" the main is "idea" in terms of correct distribution of "power" and "coordinated breathing" "inner air" is reproduced. In notion "E" controls "TSI" with the help of the idea there is the control over "inner air" movement. For the beginners it is difficult to master the category "E" controls "TSI": first of all, organism is not so flexible, secondly, "TSI" didn't achieve harmony yet, thirdly, meridians channels are not effective enough and all work in them can be realized in great tension $[5,16]$.

\section{RESULTS AND DISCUSSION}

Individual features, time and the ways of trainings

The methodology of Chi Kung gymnastics from the point of view of its therapeutic characteristics should be considered in a complex approach, taking into account physiological aspects, pathology, theory of medicine $[2,5,12,15]$. Thus, taking into account these factors, we come to the conclusion that for those, who begin their trainings according to one of the main complexes, should take into consideration that there will be some differences for men and women and for people with different physiological indices. The demands claimed on the exercises for different diseases treatment will also be different. For the diseases of one type they will also differ. The demands claimed on the exercises will also be different at different stages of the disease (disease slow down and the period of recovery).

For example, fulfilling three respiration "tsi" using the average "Dan-tyan", both hands are placed before the average "Dan-tyan", men start with the left hand, women start with the right hand. During the exercises with sticks fulfillment (in case if manipulation is realized with one hand) men hold the stick in the left hand and women in the right hand. The exercises are created taking into account physiological gender demorphism $[1,5]$.

In some exercises the order of legs putting forward (first goes the left leg or vice versa), the way of legs placing on the ground (the leg is first placed on heel or a toe) is mentioned. During two hands lifting it is mentioned where the palms should be directed to. The speed and amplitude of body flexion and extension during respiratory exercises fulfillment is also mentioned. All recommendations are done taking into account different diseases, all corrections are fulfilled, taking into account the state of blood vessels and balance of "yin" and "yan". For example, for people with the increased function of arterial pressure (depending on the level of blood pressure and ocular tension increase, on excessive blood integrity in terms of the increased blood sugar, cholesterol, in terms of not normal indices in the analysis) and for people with the decreased function (depending on the level of blood pressure decrease, on the level of blood sugar decrease, in terms of other deviations) there are also differences in exercises fulfillment [11,12]. Thus, in every definite case for each person special methodology of exercises should be selected. It corresponds with the character of his disease.

The presented methodologies of exercises for people with different diseases provide different ways of respiratory exercises fulfillment. For example, for people with cancer or people with phlogotic processes (if they are not extremely weak) "fen" way of breathing is used [18].For other people it is better to use "TSI" way of breathing or natural breathing (especially for the patients with high blood pressure, people with cardiac defects, for whom only "TSI" or natural breathing are recommended). At the same time in "fen" way of breathing there are also some moments, which differ according to the tempo of respiratory exercises, for example, "one inhalation" - "one exhalation" or "two inhalations""one exhalation". It is also necessary to follow these methodologies in accordance with the definite recommendations $[17,18]$.

It is known that there are different diseases, which restrict the use of the definite rules [19]. Such 
a rule, which is restricted in use, is "bu-se". This rule use is strictly individual for every person. During the exercises fulfillment in order to realize "se" first of all inhalation is fulfilled, then exhalation, for "bu" realization first goes exhalation and then inhalation. During massage fulfillment "bu" of "se" rules, or neither "bu", nor "se" rules are used, but stabilizing influence. People with chronic diseases need the ways of treatment application according to "bu" rule.

During preliminary complexes creation in preparatory and final periods the rules of "inner air" reproduction, its movement and circulation should be taken into account. During the exercises fulfillment it is necessary to observe all recommendations, not changing them to your taste.

Pregnant women and women during menstruation undergo the definite physiological changes, that is why they have to be more attentive fulfilling the exercises, which influence blood and "air" circulation in an organism. Usually in these cases "fen" breathing (as this way has sedative effect on "se", during menstruations a woman shouldn't do any exercises with "se" influence) is not practiced. The complexes of exercises with "fen" breathing in this case should be changed to the exercises with natural breathing. During the massage fulfillment with the help of sticks and during lifts and lowerings in an open and close positions low squats also shouldn't be fulfilled [1,2,5,19]. During "Yumtsyuan" points massage, which are situated on the head, stabilizing actions should be fulfilled, taking into account the level of a person's functional state.

\section{CONCLUSION}

1.Chi Kung system was known since ancient times and it was closely connected with alchemical practice of self-regulation till the state of extreme long life achievement. According to some sources, Dao Yin belongs to not traditional methods of Chinese medicine. It belongs to the system of Taoistic inner regulation, the founder of which is Lao Tsy.

2.Since ancient times Chi Kung was widely used in China for medical, military, scientific, creative and moral-ethical needs. The methodologies included static, dynamic and mixed techniques according to style of movements, they also were divided into inner and outer ones, according to the quality of fulfillment- soft, severe and co-joining ones.

3.In the sphere of medicine Chi Kung differs from other therapeutic methods, adopted in Chinese medicine and this term underlines the unique character of this system.

\section{REFERENCES}

1.Chang MY. The Theory and Practice of Health Cultivation Qigong Exercise in Traditional Chinese Medicine. HuLiZaZhi. 2015 Dec;62(6):139. doi: 10.6224/JN62.6.1

2.Klein PJ, Baumgarden J, Schneider R. Qigong and Tai Chi as Therapeutic Exercise: Survey of Systematic Reviews and Meta-Analyses Addressing Physical Health Conditions. Altern Ther Health Med. 2019 Jun 1. pii: AT5817. [Epub ahead of print]

Huang J, Wang XY. Review of centennial development of Qigong. ZhonghuaYiShiZaZhi. $2012 \mathrm{Jul} ; 42(4): 201-7$.

Yang YW, Wu HZ. Origin and development of qigong-wuqinxi. Zhonghua Yi Shi ZaZhi. 2011 Sep;41(5):265-7.

Butenko V.D. About the mechanism of health improvement in Chi Kung system. Sovremennye naukoemkie tehnologii. 2008;7:8 (In Russ.).

Birdee GS1, Wayne PM, Davis RB, Phillips RS, Yeh GY. T'ai chi and qigong for health: patterns of use in the United States. $J$ Altern Complement Med. 2009 Sep;15(9):969-73. doi: 10.1089/ acm.2009.0174.

Wang CC, Li K, Choudhury A, Gaylord S. Trends in Yoga, Tai Chi, and Qigong Use Among US Adults, 2002-2017. Am J Public Health. 2019 May;109(5):755-761. doi: 10.2105/ AJPH.2019.304998.

Ibañez GE, Algarin A, Taskin T. Letter Response: Yoga, Tai Chi, Qigong, and Health Disparities. Am J PublicHealth. 2019 Sep;109(9):e5. doi: 10.2105/AJPH.2019.305238.

Tarabrina N.Yu., Kraev Yu.V., Tikhonov A.I. Study of the effect of «Qigong» on the functional state of cardiorespiratory system of schoolchildren. Proceedings of the 1st International Volga Region 
Conference on Economics, Humanities and Sports «FICEHS 2019» Kazan, Naberezhnye Chelny. 2019: 223-225.

Vong Kyu Kit. Tsigung I zdorov'e [Chi Kung and health]. Saint-Petersburg: The future of the Earth. 2006; 239 (In Russ.).

Luvsan G.V. About the traditions of eastern medicine. Complex of not difficult effective exercises. Sportivnaya zhizn' Rossii.2011;7:29-30 (In Russ.).

Tarabrina N.Yu. Miorelaxation in the correction system of scoliotic changes of thoracic spine of athletes. Teoriya I praktika fizicheskoj kul'tury = Theory and practice of physical culture. 2016;4:30-32 (In Russ., In Engl.).

Guo Y, Shi H, Yu D, Qiu P. Health benefits of traditional Chinese sports and physical activity for older adults: A systematic review of evidence. $J$ Sport Health Sci. 2016 Sep;5(3):270-280. doi: 10.1016/j.jshs.2016.07.002.

Shin SS. Development of integrated traditional Chinese and western medicine and change of medical policy in China. Uisahak. 1999;8(2):207-32.

Tarabrina N.Yu. The effectiveness of rhythmic gymnastics estimation in functional state correction of students with obesity. Kul'tura fizicheskaya I zdorov'e.. 2017;1(61):100-102.
Belova L.B. Tsigung: 7 shelkovyh dvizhenij zdorov'ya, molodosti I dolgoletiya [Chi Kung: 7 silk movements of health, youth and long life]. Moscow: AST Saint-Petersburg. Sova. 2008: 158.

Chen KW. An analytic review of studies on measuring effects of external QI in China.AlternTher Health Med. 2004 Jul-Aug;10(4):38-50.

Zeng Y, Xie X, Cheng ASK. Qigong or Tai Chi in Cancer Care: an Updated Systematic Review and Meta-analysis. CurrOncolRep. 2019 Apr 6;21(6):48. doi: 10.1007/s11912-019-0786-2.

Jerger KK, Lundegard L, Piepmeier A, Faurot K, Ruffino A, Jerger MA, Belger A. Neural Mechanisms of Qigong Sensory Training Massage for Children With Autism Spectrum Disorder: A Feasibility Study. GlobAdvHealthMed. 2018 Apr 5;7:2164956118769006. doi: 10.1177/2164956118769006

Kim-Kimen A.N., Kuznetsova Z.M. The development of the ancestors traditions for the Yakut sports. Pedagogiko-psihologicheskie I medico-biologicheskie problemy fizicheskoj kul'tury I sporta = The Russian Journal of Physical Education and Sport. 2018; 13(2): 143-148. DOI: 10.14526/02_2018_319 (In Russ., In Engl.).

\section{Submitted: 03.11.2019}

\section{Author's information:}

Natalya Yu. Tarabrina - Candidate of Biological Science, Moscow Aviation Institute (National Research University), 125993, Russia, Moscow, A-8o, GSP-3, Volokolamsk highway, House 4, e-mail: nata-tarabrina@mail.ru

Yuriy V. Kraev - Candidate of Psychological Sciences, Moscow Aviation Institute (National Research University), 125993, Russia, Moscow, A-8o, GSP-3, Volokolamsk highway, House 4, e-mail: yury.kraev@ mail.ru 